International Journal of Pure and Applied Mathematics

Volume 93 No. $6 \quad 2014,789-798$

ISSN: 1311-8080 (printed version); ISSN: 1314-3395 (on-line version)

url: http://www.ijpam.eu

doi: http://dx.doi.org/10.12732/ijpam.v93i6.4

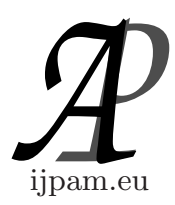

\title{
GENERAL UNIONS OF SUNDIALS (OR LINES) \\ IMPROVE THE HILBERT FUNCTIONS \\ OF PROJECTIVE SCHEMES
}

\author{
E. Ballico \\ Department of Mathematics \\ University of Trento \\ 38123 Povo (Trento) - Via Sommarive, 14, ITALY
}

\begin{abstract}
A sundial $T \subset \mathbb{P}^{r}$ is a certain flat limit of two disjoint lines with $T_{\text {red }}$ a reducible conic. Let $A \subset \mathbb{P}^{r}, r$ large, the double of a linear space. We prove that a general union of $A$ and lines or sundials has the expected postulation. Instead of $A$ we may take other low dimensional multiple structures if certain numerical conditions on their Hilbert function are satisfied.
\end{abstract}

AMS Subject Classification: 14 N05

Key Words: sundial, lines, postulation, Hilbert function

\section{Introduction}

For any reduced closed subscheme $W$ of a reduced scheme $X$ and any integer $m>0$ let $(m W, X)$ denote the $(m-1)$-th infinitesimal neighborhood of $W$ in $X$, i.e. the closed subscheme of $X$ with $\left(\mathcal{I}_{W, X}\right)^{m}$ as its ideal sheaf. We often write $m W$ if $X=\mathbb{P}^{r}$.

For any integer $x \in\{0, \ldots, r-1\}$ let $V_{x, r} \subset \mathbb{P}^{r}$ be an $x$-dimensional linear subspace. See Lemma 1 for the well-known description of the Hilbert polynomial of $m V_{x, r}$ and Lemma 4 for its Hilbert function. A sundial $A \subset \mathbb{P}^{r}$ is a closed subscheme such that $A_{\text {red }}$ is a reducible conic, $T$, and there is a 3 -

Received: February 22, 2014

(c) 2014 Academic Publications, Ltd. url: www.acadpubl.eu 
dimensional linear linear space $V \subseteq \mathbb{P}^{r}$ such that $A=A \cup(2 P, V)([2])$. $A$ is a flat limit of a family of pairs of disjoint lines ([4]). A closed subscheme $X \subset \mathbb{P}^{r}$ is said to have maximal rank if for every integer $t>0$ either $h^{1}\left(\mathcal{I}_{X}(t)\right)=0$ or $h^{0}\left(\mathcal{I}_{X}(t)\right)=0$. A general union of lines has maximal rank ([4]). A general union of sundials and lines has maximal rank ([2]). Adding a general union of many disjoint lines in $\mathbb{P}^{r}, r \geq 4$, to a zero-dimensional scheme we get a scheme with maximal rank ([1]). In a few cases (e.g, for $m V_{r, 0}, r \geq 4$ and $2 V_{0,3}$ ([3]), [1], Proposition 3, for a cohomologically similar case), this is true for an arbitrary number of general disjoint lines. In the case $r \geq 4$ it is easy to extend it to general unions of sundials and lines (see Proposition 1). We prove the following results.

Theorem 1. Fix integers $r \geq 5, t \geq 0$ and $e \geq 0$. Let $X \subset \mathbb{P}^{r}$ be a general union of $2 V_{1, r}, t$ sundials and $e$ lines. Then $X$ has maximal rank.

Theorem 2. Fix integers $x \geq 2, r \geq 2 x+4, t \geq 0$ and $e \geq 0$. Let $X \subset \mathbb{P}^{r}$ be a general union of $2 V_{x, r}, t$ sundials and $e$ lines. Then $X$ has maximal rank.

We prove these results checking the numerical inequality coming in the following statement.

Theorem 3. Fix an integers $r \geq 4, m>0$ and a closed subscheme $Z \subset \mathbb{P}^{r}$ such that $\operatorname{dim}(Z) \leq r-2, h^{0}\left(\mathcal{I}_{Z}(m-1)\right)=0$ and $h^{i}\left(\mathcal{I}_{Z}(t)\right)=0$ for all $t \geq m-i$ and all $i>0$. Let $H \subset \mathbb{P}^{r}$ be a general hyperplane section. Assume $h^{0}\left(H, \mathcal{I}_{Z \cap H}(m-1)\right)=0$ and $h^{1}\left(H, \mathcal{I}_{Z \cap H}(t)\right)=0$ for all $i>0$ and $t \geq m-1$. Set $f_{Z}(t):=h^{0}\left(\mathcal{O}_{Z}(t)\right)$ and $\left.f_{Z \cap H}(t)\right):=h^{0}\left(\mathcal{O}_{Z \cap H}(t)\right)$. Assume that for all $a^{\prime} \geq 0$ and $b^{\prime} \geq 0$ a general union $Y \subset H$ of $Z \cap H$, $a^{\prime}$ sundials and $b^{\prime}$ lines have maximal rank. Assume

(a) $\left.\begin{array}{c}r+k-1 \\ r-1\end{array}\right) k(r-1) / r-k f_{Z \cap H}(k)+f_{Z}(k-1)>k^{2}(k+1)$ for all $k>m$

(b) $\left(\begin{array}{c}r+k \\ r\end{array}\right) \geq(2 k+1)(k+1)+f_{Z}(k)$ for all $k \geq m$.

Then for all integers $\alpha \geq 0$ and $\beta \geq 0$ a general union $X \subset \mathbb{P}^{r}$ of $Z, \alpha$ sundials and $\beta$ lines have maximal rank.

We think that the inequalities in (a) and (b) hold for $Z=m V_{x, r}$ for arbitrary $m$ and $x$ if $r \gg m$ and $r \gg x$. To apply Theorem 3 need weaker numerical assumptions than a corresponding statements we could prove using using only lines and a few reducible conics as in [4] instead of using sundials. 


\section{The schemes $m V_{x, r}$}

For any line bundle $\mathcal{L}$ and any integer $a>0$ we often write $a \mathcal{L}$ as a short-hand of $\mathcal{L}^{\oplus a}$.

Lemma 1. Fix integers $r>x \geq 0$ and $m>0$. Then

$$
\mathcal{O}_{m V_{x, r}} \cong \oplus_{i=0}^{m-1}\left(\begin{array}{c}
r-x+i-1 \\
i
\end{array}\right) \mathcal{O}_{V_{x, r}}(-i)
$$

as $\mathcal{O}_{V_{x, r}}$-sheaves.

Proof. We use induction on $m$, the case $m=1$ being obvious. Assume $m \geq 2$ and that the lemma is true (for all $x, r$ ) for all multiplicities $m^{\prime}<m$. Set $\mathcal{I}:=\mathcal{I}_{V_{x, r}}$. The $\mathcal{O}_{V_{x, r}}$ sheaf $\mathcal{I} / \mathcal{I}^{2}$ is isomorphic to the conormal bundle of $V_{x, r}$ in $\mathbb{P}^{r}$. Hence $\mathcal{I} / \mathcal{I}^{2} \cong(r-x) \mathcal{O}_{V_{x, r}}(-1)$. Look at the following exact sequence of $\mathcal{O}_{V_{x, r}}$ sheaves:

$$
0 \rightarrow \mathcal{I}^{m} \rightarrow \mathcal{I}^{m-1} \rightarrow \mathcal{I}^{m-1} / \mathcal{I}^{m} \rightarrow 0
$$

Since $\mathcal{I}^{m-1} / \mathcal{I}^{m} \cong S^{m}\left(\mathcal{I} / \mathcal{I}^{2}\right) \cong\left(\begin{array}{c}r-x+m-1 \\ m\end{array}\right) \mathcal{O}_{V_{x, r}}(-m)$ as $\mathcal{O}_{V_{x, r} \text {-sheaves, }}$ (1) and the inductive assumption gives that $\mathcal{O}_{m V_{x, r}}$ is isomorphic as an $\mathcal{O}_{V_{x, r}}$-sheaf to an extension of the vector bundle $\left(\begin{array}{r}r-x+m-1 \\ m\end{array}\right) \mathcal{O}_{V_{x, r}}(-m)$ by the vector bundle

$$
\oplus_{i=0}^{m-2}\left(\begin{array}{c}
r-x+i-1 \\
i
\end{array}\right) \mathcal{O}_{V_{x, r}}(-i)
$$

If $x \neq 1$, then this extension splits. Now assume $x=1$. We apply the lemma to $m V_{2, r+1}$ and then take the intersection of $m V_{2, r+1}$ with a general hyperplane of $\mathbb{P}^{r+1}$.

Lemma 2. We have $h^{1}\left(\mathcal{I}_{m V_{x, r}}(m)\right)=0$.

Proof. The lemma is obvious if $m=1$. Hence we may assume $m \geq 2$ and use induction on $m$. The lemma is true if $x=r-1$. Hence we may assume $r-x \geq 2$ and that the lemma is true for all integers $r^{\prime}, x^{\prime}, m^{\prime}$ with $r^{\prime}-x^{\prime}<r-x$. Fix a hyperplane $M \subset \mathbb{P}^{r}$ such that $M \supseteq V_{x, r}$. Since $\operatorname{Res}_{M}\left(m V_{x, r}\right)=(m-1) V_{x, r}$ and $M \cap m V_{x, r} \cong m V_{x, r-1}$, we have an exact sequence

$$
0 \rightarrow \mathcal{I}_{(m-1) V_{x, r}}(m-1) \rightarrow \mathcal{I}_{m V_{x, r}}(m) \rightarrow \mathcal{I}_{m V_{x, r-1}, M}(m) \rightarrow 0
$$

Use (2) and the inductive assumption on the integers $m$ and $x-r$.

Lemma 3. Fix integers $r>x \geq 0, m>0$. Then: 
(a) $h^{0}\left(\mathcal{I}_{m V_{x, r}}(t)\right)=0$ if and only if $t \leq m-1$.

(b) $h^{1}\left(\mathcal{I}_{m V_{x, r}}(t)\right)=0$ for all $t \geq m-1$.

(c) $h^{i}\left(\mathcal{I}_{m V_{x, r}}(t)\right)=0$ for all $i \geq 2$ and all $t \geq m$.

Proof. Part (a) is obvious. The case $x=0$ of the lemma is trivial. Assume $x>0$ and that part (b) is true for the integer $x-1$ in an $(r-1)$-dimensional projective space. Let $H \subset \mathbb{P}^{r}$ be a general hyperplane. We have $H \cap m V_{x, r}=$ $\left(m\left(H \cap V_{x, r}\right), H\right)$ (as schemes). Look at the exact sequence

$$
0 \rightarrow \mathcal{I}_{m V_{x, r}}(t-1) \rightarrow \mathcal{I}_{m V_{x, r}}(t) \rightarrow \mathcal{I}_{\left(m\left(V_{x, r} \cap H\right), H\right)}(t) \rightarrow 0
$$

Since $h^{0}\left(H, \mathcal{I}_{\left(m\left(V_{x, r} \cap H\right), H\right)}(m-1)\right)=0$ and $h^{1}\left(H, \mathcal{I}_{\left(m\left(V_{x, r} \cap H\right), H\right)}(t)\right)=0$ for all $t \geq m-1$ by the inductive assumption, for all $t \geq m$ the maps $H^{1}\left(\mathcal{I}_{m V_{x, r}}(t-\right.$ $1)) \rightarrow H^{1}\left(\mathcal{I}_{m V_{x, r}}(t)\right)$ are surjective. Lemma 2 gives $h^{1}\left(\mathcal{I}_{m V_{x, r}}(m)\right)=0$. Hence $h^{1}\left(\mathcal{I}_{m V_{x, r}}(t)\right)=0$ for all $t \geq m$. Since every element of $\left.\mid \mathcal{I}_{m V_{x, r}}(m)\right) \mid$ is a cone with vertex containing $V_{x, r}$, the restriction map

$$
H^{0}\left(\mathcal{I}_{m V_{x, r}}(m)\right) \rightarrow H^{0}\left(\mathcal{O}_{\left.\left.m V_{x, r} \cap H\right), H\right)}(m)\right)
$$

is surjective. Hence $h^{1}\left(\mathcal{I}_{m V_{x, r}}(m-1)\right) \leq h^{1}\left(\mathcal{I}_{m V_{x, r}}(m)\right)=0$. For all $i \geq 2$ we have $h^{i}\left(\mathcal{I}_{m V_{x, r}}(t)\right)=h^{i-1}\left(\mathcal{O}_{m V_{x, r}}(t)\right)$ and the latter integer is zero if either $i \geq x+2$ or $i \leq x$ or $i=x+1$ and $t \geq m-x+1$ by Lemma 1 .

Remark 1. For all integers $r>x>0, t>m>0$ we have $h^{0}\left(\mathcal{I}_{m V_{x, r}}(t)\right)=$ $h^{0}\left(\mathcal{I}_{m V_{x, r}}(t-1)\right)+h^{0}\left(\mathcal{I}_{m V_{x-1, r-1}}(t)\right)$ and $h^{0}\left(\mathcal{O}_{m V_{x, r}}(t)\right)=h^{0}\left(\mathcal{O}_{m V_{x, r}}(t-1)\right)+$ $h^{0}\left(\mathcal{O}_{m V_{x-1, r-1}}(t)\right)$ and $h^{0}\left(\mathcal{I}_{m V_{x, r}}(t)\right)=h^{0}\left(\mathcal{I}_{m V_{x, r}}(t-1)\right)+h^{0}\left(\mathcal{I}_{m V_{x-1, r-1}}(t)\right)$ (use (3)). From Lemma 1 we get

$$
h^{0}\left(\mathcal{O}_{m V_{x, r}}(t)\right)=\sum_{i=0}^{m-1}\left(\begin{array}{c}
r-x+i-1 \\
i
\end{array}\right)\left(\begin{array}{c}
x+t-i \\
x
\end{array}\right)
$$

or all $t \geq m$.

Lemma 4. Fix integers $r>x \geq 0$ and $m>0$. We have $h^{0}\left(\mathcal{I}_{m V_{x, r}}(t)\right)=0$ if $t \leq m-1$ and $h^{0}\left(\mathcal{I}_{m V_{x, r}}(t)\right)=\left(\begin{array}{c}r+t \\ r\end{array}\right)-\sum_{i=0}^{m-1}\left(\begin{array}{c}r-x+i-1 \\ i\end{array}\right)\left(\begin{array}{c}x+t-i \\ x\end{array}\right)$ if $t \geq m$.

Proof. Apply Remark 1 and parts (a) and (b) of Lemma 3. 


\section{The proofs}

For all integers $r \geq x+3 \geq 3$ and $k \geq m>0$ define the integers $a_{r, x, m, k}$ and $b_{r, x, m, k}$ by the relations

$$
(k+1) a_{r, x, m, k}+b_{r, x, m, k}=\left(\begin{array}{c}
r+k \\
r
\end{array}\right)-h^{0}\left(\mathcal{O}_{m V_{x, r}}(k)\right), 0 \leq b_{r, x, m, k} \leq k
$$

For the values of the integers $h^{0}\left(\mathcal{O}_{m V_{x, r}}(k)\right)$, see Remark 1. If $x>0$ and $k>m$, then Remark 1 and (1) for the integers $k$ and $k-1$ give the following equality

$$
\begin{aligned}
& a_{r, x, m, k-1}+(k+1)\left(a_{r, x, m, k}-a_{r, x, m, k-1}\right)+b_{r, x, m, k}-b_{r, x, m, k-1} \\
& =\left(\begin{array}{c}
r+k-1 \\
r-1
\end{array}\right)-h^{0}\left(\mathcal{O}_{m V_{x-1, r-1}}(k)\right)
\end{aligned}
$$

The equality (5) is true also if $x=0$, just setting $h^{0}\left(\mathcal{O}_{m V_{-1, r-1}}(k)\right)=0$ (i.e. taking $V_{-1, n}=\emptyset$ for any $\left.n>0\right)$.

Lemma 5. Fix integers $x \geq 0, r \geq 4+x, m>0, t \geq 0$ and $e \geq 0$. Let $W \subset \mathbb{P}^{r}$ be a general union of $t$ sundials and $e$ lines. Set $X:=V_{x, r} \cup W$. Then either $h^{0}\left(\mathcal{I}_{X}(m)\right)=0$ or $h^{1}\left(\mathcal{I}_{X}(m)\right)=0$.

Proof. Let $\ell: \mathbb{P}^{r} \backslash V_{x, r} \rightarrow \mathbb{P}^{r-x-1}$ be the linear projection from $V_{x, r}$. Since $W$ is general and $r \geq x+4$, we have $V_{x, r} \cap W=\emptyset$ and $\ell(W)$ is a general disjoint union of $t$ sundials and $e$ lines. Since $r-x-1 \geq 3, \ell(W)$ has maximal $\operatorname{rank}([2])$. Hence either $h^{0}\left(\mathbb{P}^{r-x-1}, \mathcal{I}_{\ell(W)}(k)\right)=0$ or $h^{1}\left(\mathbb{P}^{r-x-1}, \mathcal{I}_{\ell(W)}(k)\right)=$ 0 . Since $\left|\mathcal{I}_{m V_{x, r}}(m)\right|$ is the set of all degree $m$ cones with vertex containing $V_{x, r}$, we have $h^{0}\left(\mathcal{I}_{X}(m)\right)=h^{0}\left(\mathbb{P}^{r-x-1}, \mathcal{I}_{\ell(W)}(k)\right)$. We also have $h^{1}\left(\mathcal{I}_{X}(m)\right)=$ $h^{1}\left(\mathbb{P}^{r-x-1}, \mathcal{I}_{\ell(W)}(k)\right)$ by part (b) of Lemma 3.

Proposition 1. Fix integers $r \geq 4, m>0, t \geq 0$ and $e \geq 0$. Let $X \subset \mathbb{P}^{r}$ be a general union of $m V_{0, r}, t$ sundials and $e$ lines. Then $X$ has maximal rank.

Proof. By Lemma 4 we may assume $(t, e) \neq(0,0)$. Since a general union of a prescribed number of lines and sundials and one point has maximal rank ([2]), it is sufficient to do the case $m \geq 2$. Since a sundial is a flat limit of a family of disjoint lines ([4]), it is sufficient to do the case $e=0$ and the case $e=1$. Let $k$ be the minimal positive integer such that $\left(\begin{array}{c}r+m-1 \\ r\end{array}\right)+(2 t+e)(k+1) \leq\left(\begin{array}{c}r+k \\ r\end{array}\right)$ (this integer is called the critical value of the triple $(r, m, 2 t+e))$. Since $2 t+e>0$ we have $k \geq m$. For the case $k=m$ see the case $x=0$ of Lemma 5 . Hence we may assume $k>m$ and that Proposition 1 is true (for fixed integers $r$ and 
$m$ ) for the pairs $\left(t^{\prime}, e^{\prime}\right)$ such that $\left(r, m, 2 t^{\prime}+e^{\prime}\right)$ has critical value $<k$. By the Castelnuovo-Mumford's lemma it is sufficient to prove that $h^{1}\left(\mathcal{I}_{X}(k)\right)=0$ and $h^{0}\left(\mathcal{I}_{X}(k-1)\right)=0$. Fix $P \in \mathbb{P}^{r}$. Since Aut $\left(\mathbb{P}^{r}\right)$ is transitivity, without losing the generality of $X$ we may assume that $\{P\}=V_{0, r}$. Let $H \subset \mathbb{P}^{r}$ be a hyperplane such that $P \notin H$.

(a) In this step we prove that $h^{1}\left(\mathcal{I}_{X}(k)\right)=0$. Increasing if necessary either $t$ or $e$ we may assume $\left(\begin{array}{c}r+m-1 \\ r\end{array}\right)+(2 t+e)(k+1) \geq\left(\begin{array}{c}r+k \\ r\end{array}\right)-k$, i.e. we may assume that $t=\left\lfloor a_{r, 0, m, k} / 2\right\rfloor$ and $e=a_{r, 0, m, k}-2 t$. Set $e^{\prime}:=a_{r, 0, m, k-1}-$ $2\left\lfloor a_{r, 0, m, k-1} / 2\right\rfloor \in\{0,1\}$. Let $Y \subset \mathbb{P}^{r}$ be a general union of $\left\lfloor a_{r, 0, m, k-1} / 2\right\rfloor$ sundials and $e^{\prime}$ lines. Since $a_{r, 0, m, k-1}>a_{r, 0, m, k-2}$ (Lemma 7), the inductive assumption gives that $h^{1}\left(\mathcal{I}_{Y}(k-1)\right)=0$ and $h^{0}\left(\mathcal{I}_{Y}(k-2)\right)=0$. Since $h^{1}\left(\mathcal{I}_{Y}(k-\right.$ $1))=0$, we have $h^{0}\left(\mathcal{I}_{Y}(k-1)\right)=b_{r, 0, m, k-1}$. Let $S \subset H$ be a general subset with $\sharp(S)=b_{r, 0, m, k-1}$. Since $h^{0}\left(\mathcal{I}_{Y}(k-2)\right)=0, \operatorname{Res}_{H}(Y)=Y$ and $h^{0}\left(\mathcal{I}_{Y}(k-1)\right)=$ $b_{r, 0, m, k-1}$, we have $h^{0}\left(\mathcal{I}_{Y \cup S}(k-1)\right)=0$. The case $x=0$ and $k^{\prime}=k-1$ of $(5)$ gives $h^{1}\left(\mathcal{I}_{Y \cup S}(k-1)\right)=0$.

(a1) First assume $e^{\prime}=e$. Let $E \subset H$ be a general union of $t-\left\lfloor a_{r, 0, m, k-1} / 2\right\rfloor$ reducible conics and write $E=E_{1} \sqcup E_{2}$ with $E_{2}$ a general union of $b_{r, 0, m, k-1}$ conics. Let $G_{1} \subset H$ be a general union of sundials with $\left(G_{1}\right)_{\text {red }}=E_{1}$. Let $G_{2} \subset \mathbb{P}^{r}$ be a general union of sundials with $\left(G_{2}\right)_{\text {red }}=E_{2}$. Set $X^{\prime}:=Y \cup G_{1} \cup G_{2}$. Since $E_{2}$ is a general union of $b_{r, 0, m, k-1}$ reducible conics contained in $H$, we may assume that $S$ is the support of the nilpotent sheaf of $G_{2}$. By the semicontinuity theorem for cohomology it is sufficient to prove that $h^{1}\left(\mathcal{I}_{X^{\prime}}(k)\right)=0$. We have $X^{\prime} \cap H=(Y \cap H) \cup G_{1} \cup E_{2}$ and $\operatorname{Res}_{H}\left(Y^{\prime}\right)=Y \cup S$. Since $h^{1}\left(\mathcal{I}_{Y \cup S}(k-1)\right)=0$, it is sufficient to prove that $\left.h^{0}\left(H, \mathcal{I}_{X^{\prime} \cap H}(k)\right)\right)=0$. We have $h^{0}\left(\mathcal{O}_{X^{\prime} \cap H}(k)\right)=a_{r, 0, m, k-1}+h^{0}\left(\mathcal{I}_{G_{1} \cup E_{2}}(k)\right)=a_{r, 0, m, k-1}+(k+1)\left(a_{r, 0, m, k}-\right.$ $\left.a_{r, 0, m, k-1}\right)-b_{r, 0, m, k-1} \leq\left(\begin{array}{c}r+k-1 \\ r-1\end{array}\right)$ by (5). Since $Y \cap H$ is a general subset of $H$ with cardinality $\sharp(Y \cap H)$, it is sufficient to prove that $h^{1}\left(H, \mathcal{I}_{G_{1} \cup E_{2}}(k)\right)=$ 0 . Let $F_{2} \subset H$ be a general union of sundials with $\left(F_{2}\right)_{\text {red }}=E_{2}$. Since $h^{1}\left(\mathcal{I}_{\operatorname{Res}_{H}\left(Y^{\prime}\right)}(k-1)\right)=0$, a Castelnuovo's sequence shows that it is sufficient to prove that $h^{1}\left(H, \mathcal{I}_{G_{1} \cup F_{2}}(k)\right)=0 . G_{1} \cup F_{2}$ is a general union of sundials. Hence by [2] it is sufficient to check that $h^{0}\left(\mathcal{O}_{G_{1} \cup F_{2}}(k)\right) \leq\left(\begin{array}{c}r+k-1 \\ r-1\end{array}\right)$. By (5) it is sufficient to use that $\sharp(Y \cap H)=a_{r, 0, m, k-1} \geq b_{r, 0, m, k-1}$. This inequality is true by Lemma 6 , because $b_{r, 0, m, k-1} \leq k-1$.

(a2) Now assume $e^{\prime}=0$ and $e=1$. Instead of $E=E_{1} \sqcup E_{2}$ we take $E=E_{1} \sqcup E_{2} \sqcup L$ with $L$ a general line of $H$. We work in $H$ as in step (a1).

(a3) Now assume $e^{\prime}=1$ and $e=0$. Write $Y=Y^{\prime} \sqcup R$ with $Y^{\prime}=m P \sqcup W$, $W$ general union of $\left(a_{r, 0, m, k-1}-1\right) / 2$ sundials and $L$ a general line. Take a general $E_{1} \sqcup E_{2} \sqcup L \subset H$ with $E_{1}$ general union of $t-\left(a_{r, 0, m, k-1}-1\right) / 2-b_{r, 0, m, k-1}$ reducible conics, $E_{2}$ a general union of $b_{r, 0, m, k-1}$ reducible conics and $L$ a general 
line of $H$ through the point $R \cap H$. For a general line $R$ the point $R \cap H$ is a general point of $H$. Hence $E_{1} \sqcup E_{2} \sqcup L$ has the Hilbert function of a general union of $t-\left(a_{r, 0, m, k-1}-1\right) / 2$ reducible conics and one line. Let $L^{\prime} \subset H$ be a general +line of $H$ with $L$ as its support and with $P$ as the support of its ideal sheaf, i.e. a general scheme $L \cup \nu \subset H$ with $\nu \subset H$ a general degree 2 scheme with $\nu_{\text {red }}=\{P\}$. Let $G_{1} \subset H$ be a general union of sundials with $\left(G_{1}\right)_{\text {red }}=E_{1}$. Let $G_{2} \subset \mathbb{P}^{r}$ be a general union of sundials with $\left(G_{2}\right)_{\text {red }}=E_{2}$. Set $X^{\prime \prime}:=Y \cup G_{1} \cup G_{2} \cup L^{\prime}$. Since $R \cup L^{\prime}$ is a sundial of $\mathbb{P}^{r}$ and $\operatorname{Res}_{H}\left(X^{\prime \prime}\right)=Y \cup S$, it is sufficient to prove that $h^{1}\left(H, \mathcal{I}_{X^{\prime \prime} \cap H}(k)\right)=0$. Let $F_{2} \subset H$ be a general union of sundials with $\left(F_{2}\right)_{\text {red }}=E_{2}$. Take a general line $D \subset H$ through $R \cap H$ and set $T:=L^{\prime} \cup D$, i.e. take a general sundial of $H$ with $R \cap H$ as the support of the nilpotent sheaf and $L$ as an irreducible component of $T_{\text {red }}$. In this step we need $a_{r, 0, m, k-1} \geq b_{r, 0, m, k-1}+k$ (see Lemma 6 ).

(b) In this step we prove that $h^{0}\left(\mathcal{I}_{X}(k-1)\right)=0$. For the case $k-1=m$ see Lemma 5. Hence we may assume $k \geq m+2$. By the definition of the integer $k$ we have $2 t+e>a_{r, 0, m, k-1}$. Since a line is contained in a sundial, it is sufficient to do the case $2 t+e=a_{3,0, m, k-1}+1$. Let $Y^{\prime} \subset \mathbb{P}^{r}$ be a general union of $\left\lfloor a_{r, 0, m, k-2} / 2\right\rfloor$ sundials and $e^{\prime \prime}:=a_{r, 0, m, k-2} / 2$ lines. Since $a_{r, 0, m, k-1}>$ $a_{r, 0, m, k-2}($ Lemma 7$)$, the inductive assumption gives that $h^{1}\left(\mathcal{I}_{Y^{\prime}}(k-2)\right)=0$ and $h^{0}\left(\mathcal{I}_{Y^{\prime}}(k-3)\right)=0$ (even if $k=m+2$, because $\left.h^{0}\left(\mathcal{I}_{m P}(m-1)\right)=0\right)$. Since $h^{1}\left(\mathcal{I}_{Y}(k-1)\right)=0$, we have $h^{0}\left(\mathcal{I}_{Y}(k-1)\right)=b_{r, 0, m, k-1}$. Let $S^{\prime} \subset H$ be a general subset with $\sharp(S)=b_{r, 0, m, k-2}$. Since $h^{0}\left(\mathcal{I}_{Y^{\prime}}(k-3)\right)=0$, $\operatorname{Res}_{H}\left(Y^{\prime}\right)=Y^{\prime}$ and $h^{0}\left(\mathcal{I}_{Y^{\prime}}(k-2)\right)=b_{r, 0, m, k-2}$, we have $h^{0}\left(\mathcal{I}_{Y^{\prime} \cup S^{\prime}}(k-2)\right)=0$. We make the construction in step (a) with minimal modifications. We need the same numerical checks.

Proofs of Theorems 1, 2, and 3. Look at the proof of Proposition 1. Take $Z, H$ and $Z \cap H$ as in Theorem 3. For all integers $k \geq m$ define the integers $u_{k}$ and $v_{k}$ by the relations

$$
f_{Z}(k)+(k+1) u_{k}+v_{k}=\left(\begin{array}{c}
r+k \\
r
\end{array}\right), 0 \leq v_{k} \leq k
$$

From (6) for the integers $k$ and $k-1$ we get

$$
f_{Z \cap H}(k)+u_{k-1}+(k+1)\left(u_{k}-u_{k-1}\right)+v_{k}-v_{k-1}=\left(\begin{array}{c}
r+k-1 \\
r-1
\end{array}\right)
$$

Fix $(t, e) \in \mathbb{N}^{2}$ and suppose you want to prove that a general union $X$ of $Z, t$ sundials and $e$ lines are maximal rank. It is sufficient to do all cases with $e \in\{0,1\}$. Let $k$ be the minimal integer such that $u_{k} \geq 2 t+e$. To prove 
that $h^{1}\left(\mathcal{I}_{X}(k)\right)=0$ it is sufficient to do it for the case $2 t+e=u_{k}$. This is assumed to be true if $k=m$. Hence we may assume $k>m$ and use induction on the integer $k$. Condition (a) of Theorem 3 implies $u_{k}-u_{k-1} \geq k+1$ for all $k>m$ (see the proof of Lemma 7). A weaker form of Condition (b) gives the inequality $u_{k-1} \geq k-1$ used at the end of step (a1) (as well silently in (a2) and (b)). Condition (b) gives the inequality $u_{k-1} \geq 2 k-1$ used in step (a3). In the set-up of Theorems 1 and 2 we have $Z=2 V_{x, r}, Z \cap H=2 V_{x-1, r-1}$, $m=2, u_{k}=a_{r, x, 2, k}$ and $v_{k}=b_{r, x, 2, k}$, with $x=1$ for Theorem 2. The case $k=m$ of Theorems 1 and 2 is true by Lemma 5 . Lemmas 9 and 10 gives that $a_{r, x, 2, k}-a_{r, x, 2, k-1} \geq k+1$. Lemma 6 gives the inequality $a_{r, x, 2, k-1} \geq 2 k-1$ needed to carry over step (a3).

Lemma 6. For all integers $r \geq 4$ and $t \geq m \geq 2$ we have $a_{r, 0, m, t} \geq 2 t+1$.

Proof. Assume $a_{r, 0, m, t} \leq 2 t$. We get $(2 t+1) t+\left(\begin{array}{c}m+r-1 \\ r\end{array}\right) \geq 1+\left(\begin{array}{c}r+t \\ r\end{array}\right)$. Set $v(r, t, m):=\left(\begin{array}{c}r+t \\ r\end{array}\right)-\left(\begin{array}{c}r+m-1 \\ r\end{array}\right)-2 t^{2}-t$. It is sufficient to prove that $v(r, t, m) \geq 0$. We have $v(r, m, m)=\left(\begin{array}{c}r+m-1 \\ r-1\end{array}\right)-2 m^{2}-m \geq 0$ with equality if and only if $(r, m) \in$ $\{(4,2),(4,3)\}$. We have $w(r, t):=\left(v(r, t+1, m)-v(r, t, m)=\left(\begin{array}{c}r+t \\ r-1\end{array}\right)-4 t-3 \geq 0\right.$ for all $r \geq 4$ and all $t \geq 2$.

Lemma 7. Fix integers $r \geq 4$ and $k \geq m \geq 2$. Then $a_{r, 0, m, k}-a_{r, 0, m, k-1} \geq$ $k+1$.

Proof. We first check the case $k=m$. Notice that $a_{r, 0, m, m-1}=0$ and $a_{r, 0, m, m}=\left\lfloor\left(\begin{array}{c}r+m-1 \\ r-1\end{array}\right) /(m+1)\right\rfloor$. Hence for fixed $m$ the integer $a_{r, 0, m, m}$ is an increasing function of $m$. We have $a_{4,0, m, m}=(m+3)(m+2) / 6$ if $m \equiv 0,1$ $(\bmod 3)$ and $a_{4,0, m, m}=\left(m^{2}+5 m-2\right) / 6$ if $m \equiv 0(\bmod 3)$. Hence $a_{4,0,2,2} \geq$ $m+1$ and $a_{4,0, m, m} \geq m$ if and only if $m \geq 6$.

From now on we assume $k>m$. Assume $a_{r, 0, m, k}-a_{r, 0, m, k-1} \leq 2 k$. From (5) we get

$$
a_{r, 0, m, k-1}+(k+1) k+b_{r, 0, m, k}-b_{r, 0, m, k-1} \geq\left(\begin{array}{c}
r+k-1 \\
r-1
\end{array}\right)
$$

Multiplying it by $k$, using (5), that $k\left(\begin{array}{c}r+k-1 \\ r-1\end{array}\right)-\left(\begin{array}{c}r+k-1 \\ r\end{array}\right)=\left(\begin{array}{c}r+k-1 \\ r-1\end{array}\right) k(r-1) / r$, that $b_{r, 0, m, k} \leq k$ and that $b_{r, 0, m, k-1} \geq 0$ we get

$$
k^{2}(k+1)+k^{2} \geq\left(\begin{array}{c}
r+k-1 \\
r-1
\end{array}\right) k(r-1) / r+\left(\begin{array}{c}
r+m-1 \\
r
\end{array}\right)
$$

Call $e(r, k, m)$ the difference between the right hand side and the left hand side of $(8)$ and write $f(r, k):=e(r, k, m)-\left(\begin{array}{c}r+m-1 \\ r\end{array}\right)$. To prove the lemma for the 
triple $(r, m, k)$ it is sufficient to prove that $e(r, k, m)>0$. Hence it is sufficient to prove that $f(r, k)>0$. For a fixed integer $k$ the function $f(r, k)$ is an increasing function of $r$. We have $f(5, k) / k=(k+4)(k+3)(k+2)(k+1) / 32-k^{2}-2 k>0$ for all $k>2$. We have $f(4, k) / k=(k+3)(k+2)(k+1) / 8-k^{2}-2 k>0$ for all $k \geq 5$. Now assume $k \leq 4$. We need to check the cases $(r, m, k) \in$ $\{(4,2,3),(4,2,4),(4,3,4)\}$. We have $a_{4,0,2,2}=3, a_{4,0,2,3}=7, a_{4,0,2,4}=13$, $a_{4,0,3,3}=5$, and $a_{4,0,3,4}=11$. In all cases we have $a_{r, 0, m, k} \geq a_{r, 0, m, k-1}+k+1$.

Lemma 8. For all integers $r \geq x+4 \geq 5$ and $t \geq m \geq 2$ we have $a_{r, x, m, t} \geq 2 t+1$.

Proof. It is easy to check that $a_{r, x, m, k} \geq a_{r-x, 0, m, k}$. Apply Lemma 6 .

Lemma 9. Fix integers $r \geq 2 x+4 \geq 8$ and $k \geq 3$. Then $k^{2}(k+2)<$ $\left(\begin{array}{c}r+k-1 \\ r-1\end{array}\right) k(r-1) / r-k\left(\begin{array}{c}x+k-1 \\ x-1\end{array}\right)-k(r-x)\left(\begin{array}{c}x+k-2 \\ x-1\end{array}\right)+\left(\begin{array}{c}x+k-1 \\ x\end{array}\right)+(r-x)\left(\begin{array}{c}x+k-2 \\ x\end{array}\right)$.

Proof. Since the right hand side of the inequality in the lemma is $k$ times $\left(\begin{array}{c}r+k-1 \\ r-1\end{array}\right)(r-1) / r+\left(\begin{array}{c}x+k-1 \\ x\end{array}\right)(x-1) / x-(r-x)\left(\begin{array}{c}x+k-2 \\ x-1\end{array}\right)(x-2) /(x-1)$, it is sufficient to prove the inequality

$$
\begin{aligned}
& k(k+2)<\left(\begin{array}{c}
r+k-1 \\
r-1
\end{array}\right)(r-1) / r+ \\
& +\left(\begin{array}{c}
x+k-1 \\
x
\end{array}\right)(x-1) / x-(r-x)\left(\begin{array}{c}
x+k-2 \\
x-1
\end{array}\right)(x-2) /(x-1)
\end{aligned}
$$

This inequality is obvious if $r \geq 2 x+4$.

Lemma 10. For all integers $r \geq 5$ and $k \geq 3$ we have $k(k+2)<$ $\left(\begin{array}{c}r+k-1 \\ r-1\end{array}\right) k(r-1) / r+r(k-1)+1-r k$.

Proof. Set $u(r, k):=\left(\begin{array}{c}r+k-1 \\ r-1\end{array}\right) k(r-1) / r-r+1-k(k+2)$. It is sufficient to prove that $u(r, k)>0$. We have $u(5, k)=(k+4)(k+3)(k+2)(k+1) / 32-$ $4-k(k+2)>0$ for all $k \geq 3$. Then we use that $u(r+1, k) \geq u(r, k)$ for all $k \geq 4$ and that $u(r, 3)=(r+2)(r+1)(r-1) / 6-r-15>0$ for all $r \geq 5$.

\section{Acknowledgements}

The author was partially supported by MIUR and GNSAGA of INdAM (Italy). 


\section{References}

[1] E. Ballico, Postulation of a union $X \subset \mathbb{P}^{r}, r \geq 4$, of a given zerodimensional scheme and several general lines, ISRN Geometry Volume 2013, Article ID 391721, 6 pages http://dx.doi.org/10.1155/2013/391721

[2] E. Carlini, M. V. Catalisano and A. V. Geramita, 3-dimensional sundials, Cent. Eur. J. Math. 9 (2011), no. 5, 949-971.

[3] E. Carlini, M. V. Catalisano and A. V. Geramita, Reduced and nonreduced linear spaces: lines and points, arXiv: 1308.0796.

[4] R. Hartshorne and A. Hirschowitz, Droites en position générale dans $\mathbb{P}^{n}$, Algebraic Geometry, Proceedings, La Rábida 1981, 169-188, Lect. Notes in Math. 961, Springer, Berlin, 1982. 